\title{
ON THE DEGREE OF GENERALITY OF A CLASS OF ARITHMETICAL IDENTITIES
}

BY E. T. BELL

1. Introduction. In a recent paper, $A$ class of arithmetical identities, ${ }^{*}$ Oppenheim, in commenting on a special case ( $n$ not square) of the identity A below, which is due to Uspensky $\dagger$, states that the conditions on the function $H$ are "needlessly restrictive," and he gives a "general identity which will include such identities as these," (A, with $n$ not square, and the new identities of Uspensky which involve incomplete numerical functions). $\ddagger$ It is indeed readily seen that Oppenheim's identity B implies all of those of Uspensky. In view of the remarks quoted, it is perhaps in order to give a detailed proof, by general methods adapted to the specific functions and partitions in $\mathrm{A}, \mathrm{B}$, that Uspensky's A alone implies Oppenheim's B. The identities A, B are thus of precisely the same degree of generality.

2. The Identities. Each of the functions $H(w, u, v), F(w, u, v)$, $\phi(w, u, v)$ is finite and single-valued for integer values of $w, u, v$, and beyond this restriction and those stated in (1), (4), (5) for $H, F$ $\phi$, respectively, the functions are entirely arbitrary.

A. (USPENSKY). Let

(1) $H(w, u, v)=-H(-w, u, v)=H(w,-u,-v) ; H(0, u, v)=0$.

Then, if the $\sum$ on the left of (3) refers to all integers $x, y$, z such that

$$
x^{2}+y z=n, \quad y>0, \quad z>0,
$$

where $n$ is an arbitrary constant integer,

* Quarterly Journal of Mathematics, Oxford Series, vol. 2, No. 7, 1931, pp. 300-303.

$\dagger$ First published, apparently, in Kharkof Mathematical Society Communications, (2), vol. 15 (1915-1917), pp. 81-147, (Russian). See Jahrbuch über die Fortschritte der Mathematik, vol. 48, p. 1350.

$\ddagger$ This Bulletin, vol. 36 (1930), pp. 743-754. Oppenheim, loc. cit., p. 230, corrects the misprints. The considerations of the present note apply also to the identities of Mordell, which Oppenheim cites. 
(3) $\sum[H(y+z, z-y, z)-2 H(z-2 x, 2 x+2 y-z, x+y)]$ $=\epsilon(n) \sum_{j=1}^{2 m-1}[H(2 m, 2 m-2 j, m-j)-2 H(2 m-j, 2 m-j, m)]$, where $\epsilon(n)=1$ or 0 according as $n$ is or is not square, and $m \equiv\left|n^{1 / 2}\right|$.

B. (OPPENHEIM). Let

(4) $F(w, u, v)+F(w,-u,-v)$

$$
+F(-w, u, v)+F(-w,-u,-v)=0,
$$

Then

(6) $\sum F(y+z, z-y, x)=\sum[F(\xi, \eta, \zeta)+F(\xi,-\eta,-\zeta)]$, where $\sum$ on the left refers to all integers $x, y, z$ such that (7) $\phi(y+z, z-y, x)=N, y>0, z>0, \mu(z-y) \neq \nu x$,

where $N$ is an arbitrary (real or complex) constant number, $\mu, \nu$ are any real constant numbers, and $\sum$ on the right refers to all integers $\xi, \eta, \xi$ such that

(8) $\phi(\xi, \eta, \zeta)=N, 0 \neq \xi+\eta \equiv 0 \bmod 2, \xi>\eta, \mu \eta>\nu \zeta$.

If $\phi=N$ has an infinity of solutions, $F$ is assumed to be such that the infinite series concerned are absolutely convergent.

In a previous note* I showed that A implies and is implied by the identity

$$
\begin{gathered}
\left|\begin{array}{lll}
a_{3} & b_{3} & c_{3} \\
a_{3} & b_{3} & c_{3} \\
a_{2} & b_{2} & c_{2}
\end{array}\right|=0, \\
a_{j}=\theta_{j}(2 x+y+2 x), \quad b_{j}=\theta_{j}(2 x-y-2 z), \quad c_{j}=\theta_{j}(y) .
\end{gathered}
$$

The connection of this and the present note with the seriest on Quadratic partitions in course of publication in this Bulletin is indicated below, in $\$ 4$.

3. Implication of $\mathrm{B}$ by $\mathrm{A}$. By the definition of $H$, we may replace $H$ in (3) by $H_{1}$, where $H_{1}(w, u, v)$ is any function which

* This Bulletin, vol. 32 (1926), pp. 682-688, especially p. 685.

$\dagger$ See, for example, this Bulletin, vol. 37, pp. 870-875. 
is single-valued and finite for integer values of $w, u, v$, and which satisfies the parity conditions (1). We shall take

$$
H_{1}(w, u, v) \equiv \Phi_{N}(w, u, v) \Psi_{\mu, v}(u, v) H(w, u, v),
$$

where, $\Phi_{N}(w, u, v)=1$ or else $=0$, according as $\phi(w, u, v)=N$ or $\phi(w, u, v) \neq N(N$ as in $\mathrm{B})$, and where $\Psi_{\mu, \nu}(u, v)=1$ or 0 according as $\mu u-\nu v \neq 0$ or $\mu u-\nu v=0$. This choice is permissible, since

$$
\begin{aligned}
\Phi_{N}(w, u, v) & =\Phi_{N}(-w, u, v)=\Phi_{N}(w,-u,-v), \\
\Psi_{\mu, \nu}(u, v) & =\Psi_{\mu, \nu}(-u,-v),
\end{aligned}
$$

as is evident from the definition of the functions. Having made the above substitution in $\mathrm{A}$, we then replace $H(w, u, v)$ in the resulting identity (or at once in (9), before making the substitution), by its instance $F(w, u, v)+F(w,-u,-v)$, where $F$ is as in (4). Thus A implies

$$
\begin{aligned}
& \sum_{1}[F(y+z, z-y, x)+F(y+z,-z+y,-x)] \\
- & 2 \sum_{2}[F(z-2 x, 2 x+2 y-z, x+y) \\
+ & F(z-2 x,-2 x-2 y+z,-x-y)] \\
= & \epsilon(n) \sum_{3}[F(2 m, 2 m-2 j, m-j)+F(2 m,-2 m+2 j,-m+j)] \\
- & 2 \epsilon(n) \sum_{4}[F(2 m-j, 2 m-j, m) \\
+ & F(2 m-j,-2 m+j,-m)],
\end{aligned}
$$

where the limits $j=1$ to $j=2 m-1$ are to be supplied to $\sum_{3}, \sum_{4}$, and $\sum_{i}$ refers in an obvious way to all the simultaneous integer solutions of (2) and (11.i),

$$
\phi(y+z, z-y, x)=N, \quad \mu(z-y) \neq \nu x ;
$$

(11.2) $\phi(z-2 x, 2 x+2 y-z, x+y)=N, \mu(2 x+2 y-z) \neq \nu(x+y)$;

$$
\begin{aligned}
\phi(2 m, 2 m-2 j, m-j) & =N, \quad \mu(2 m-2 j) \neq \nu(m-j) ; \\
\phi(2 m-j, 2 m-j, m) & =N, \quad \mu(2 m-j) \neq \nu m .
\end{aligned}
$$

By (2), (5), (11.1) the $\sum_{1}$ term in (10) is equal to

$$
2 \sum_{1} F(y+z, z-y, x) \text {. }
$$


By (5), if $(w, u, v)=(W, U, V)$ is an integer solution of $\phi(w, u, v)=N$, then so also is $(a W, b U, b V)$, where each of $a, b$ is either of $1,-1$, and for any definite $(a, b)$ as $(w, u, v)$ runs over all solutions, so also does $(a W, b U, b V)$. Apply this to (11.3), (11.4) with $(a, b)=(-1,1)$. Then $\sum_{i}$ in $(10)$, for $i=3,4$, is half what $\sum_{i}$ becomes when $F(w, u, v)$ is replaced by $F(w, u, v)$ $+F(-w, u, v)$. Hence, by (4),

$$
\sum_{3}=0, \quad \sum_{4}=0 .
$$

Consider now the set $S$ of all solutions $(x, y, z)$ of $(7)$, and let $S_{n}$ be that subset of $S$ which contains all those solutions, and only those, which satisfy (2). Then $S_{p}, S_{q}$ are mutually exclusive if $p \neq q$, and $S$ is the logical sum of $S_{n}(n=1,2, \cdots)$. Any particular $S_{n}$ may be the null set. In (10) take $n=1,2, \cdots$, and add corresponding members of all the resulting identities. Then, since (10) is subject to (2), (11.1)-(11.4), it follows by the above and (12), (13) that $\mathrm{A}$ implies

$$
\begin{aligned}
& \sum F(y+z, z-y, x) \\
= & \sum[F(z-2 x, 2 x+2 y-z, x+y) \\
& +F(z-2 x,-2 x-2 y+z,-x-y)],
\end{aligned}
$$

where $\sum$ in the first line refers to $(7)$, and $\sum$ in the second line to (11.2) with the additional conditions $y>0, z>0$.

To reduce (14) to the form (B), we make the substitution

$$
(z-2 x, 2 x+2 y-z, x+y)=(a \xi, b \eta, b \zeta),
$$

where each of $a, b$ is a definite one of $1,-1$. Then

$$
(2 x, 2 y, z)=(2 b \zeta-a \xi-b \eta, a \xi+b \eta, 2 b \zeta-b \eta) .
$$

By the derivation of (14), it is sufficient to consider (14) subject to (2). Since $y, z$ are integers and $y>0, z>0$, we see by (16) that the right member of (14) in the case considered is equal to

$$
\sum[F(\xi, \eta, \zeta)+F(\xi,-\eta,-\zeta)],
$$

the $\sum$ referring to all integer solutions $\xi, \eta, \zeta$ of $\phi(\xi, \eta, \zeta)=N$ which are such that

$$
\begin{aligned}
& n=(b \zeta)^{2}+\frac{1}{4}(a \xi+b \eta)(a \xi-b \eta), \\
& a \xi+b \eta \equiv 0 \quad \bmod 2, \quad(18.3) \quad b(2 \zeta-\eta)>0 \text {, } \\
& \text { (18.2) } a \xi+b(\eta)>0 \text {, } \\
& \text { (18.4) } \quad b(\mu \eta-\nu \zeta) \neq 0 \text {, }
\end{aligned}
$$

for any definite $(a, b)$ as defined. 
Let us now replace (18.4) by its equivalent

$$
b(\mu \eta-\nu \zeta)>0 \text { or } b(\mu \eta-\nu \zeta)<0 .
$$

In the first of (19) take $b=1$; in the second $b=-1$. Then the sum (17) in the case being considered is half the same sum over all integers $\xi, \eta, \zeta$ such that $\phi(\xi, \eta, \zeta)=N$, subject to the sets of conditions (in the two columns)

$$
\begin{array}{rlrl}
n & =(+\zeta)^{2}+\frac{1}{4}(a \xi+\eta)(a \xi-\eta), \\
n & =(-\zeta)^{2}+\frac{1}{4}(a \xi-\eta)(a \xi+\eta), \\
a \xi+\eta & \equiv 0 \bmod 2, & a \xi-\eta \equiv 0 \bmod 2, \\
a \xi+\eta & >0, & a \xi-\eta>0, \\
2 \zeta-\eta & >0, & 2 \zeta-\eta<0, \\
\mu \eta-\nu \zeta & >0, & \mu \eta-\nu \zeta>0 .
\end{array}
$$

The conditions (20.3) may be suppressed, since the one missing possibility, $2 \zeta-\eta=0$, contributes zero to (17). This is seen as follows. If $2 \zeta=\eta$, then by (20.1), $\xi$ is even; by (20), $n$ is square, $a \xi= \pm 2 m(m$ as in A). Choose $a=1$, or $a=-1$, according as $\xi>0$, or $\xi<0$. Adding half the respective contributions to (17), we see by (4) that the total contribution vanishes.

Combining all results so far, taking $a=1$ in both columns of (20)-(20.4), and summing over both for all values of $n$, we see that A implies B with the exception that the condition $\xi+\eta \neq 0$ in (8) is here replaced by $\xi+\eta>0$. To complete the proof that A implies B it is therefore sufficient to show that if $\xi+\eta \neq 0$ be replaced by $\xi+\eta<0$ in (8), then the right of (6) vanishes. This follows by (4), since the conditions $\xi>\eta, \xi+\eta<0, \mu \eta>\nu \zeta$ are invariant under the substitution $\left(\xi^{\prime}, \eta^{\prime}, \zeta^{\prime}\right)=(-\xi, \eta, \zeta)$, where $\xi, \eta, \zeta$ are subject to the conditions (8) with $\xi+\eta \neq 0$ replaced by $\xi+\eta<0$, and since $\left(\xi^{\prime}, \eta^{\prime}, \zeta^{\prime}\right)$ runs over all solutions of $\phi(\xi, \eta, \zeta)=N$ when $(\xi, \eta, \zeta)$ does, by (5).

4. Parity Transformations. The equivalence of A, B is a simple instance of the invariance of a parity identity under a parity transformation.* Such a transformation leaves invariant the parity of the functions in the identity, or that of the given partition, or both. In this instance both are used; in (9) and in passing to (10) the transformation is on the function; in substituting

* For some account of these, see E.T. Bell, Algebraic Arithmetic, Colloquium Publications of this Society, vol. 7, 1927, and earlier papers in the Transactions of this Society, where such transformations were applied in simple cases to the arithmetic of higher forms. 
$\phi(w, u, v)$ for $w^{2}+u v$, in which $\phi(w, u, v)$ and $w^{2}+u v$ are of the same parity, it is on the partition.

If we define (as seems reasonable) the proposition $P$ to be a generalization of the proposition $Q$ when and only when $P$ implies $Q$, and $Q$ does not imply $P$, it can be proved that it is impossible to generalize a parity identity by parity transformations.* For this reason, in the notes appearing currently in this Bulletin on Quadratic partitions, the discussion is limited to such partitions and to parity functions which, so far as feasible, are unrestricted by conditions which can be introduced by parity transformations. The passage from $n$ to $N$ also is a parity transformation, since any not identically zero linear homogeneous function of functions having a given parity has the same parity.

The linear transformation (15) would be suggested naturally as a possible means of transforming $\mathrm{A}$ by that which is given by the addition theorem for the thetas used in deducing $\mathrm{A}$ from the identity stated in $\S 2$, end. All such transformations go back ultimately to the orthogonal one on four variables which makes possible Jacobi's theta formula. It can be shown (and will be discussed in another note) from theorems of Dickson $\dagger$ on certain automorphs of sums of like powers, higher than the second, that parity identities referring to partitions of degree higher than 2 not obtainable by parity transformations from quadratic partitions do not exist.

Finally it may be noticed that convergence is irrelevant if parity identities be given their most elementary significance, that of equalities between sets of vectors having parity. This was observed essentially by Glaisher, $\ddagger$ although he did not state it explicitly.

California Institute of Technology

* The like applies to the broader class of linear homogeneous transformations of the coordinates in the vector (or matric) variables of a parity function, although the parity of the transformed function, considered as a function of the new variables, is in general different from that of the function in the old variables. All such transformations amount merely to linear homogeneous transformations of the indeterminates in the trigonometric identity which is equivalent to the parity identity.

$\dagger$ Mathematische Annalen, vol. 52 (1899), pp. 561-582.

$\ddagger$ Proceedings of the London Mathematical Society, vol. 22 (1890-91), pp. 359-410. In Dickson's History, this work is reported in vol. 1, p. 310, under Sum and Number of Divisors. It might have been cited also in vol. 2, Chap.11. 